\title{
Avaliação da função sexual de mulheres jovens brasileiras durante a pandemia de SARS-CoV-2:
}

\section{Um estudo observacional}

\author{
Evaluation of sexual function of young Brazilian women during the SARS-CoV-2 pandemic: An
}

observational study

Evaluación de la función sexual de mujeres jóvenes brasileñas durante la pandemia de SARS-CoV-

\section{2: Un estudio observacional}

\author{
Magdalena Muryelle Silva Brilhante \\ ORCID: https://orcid.org/0000-0002-4246-4351 \\ Universidade Federal do Rio Grande do Norte, Brasil \\ E-mail: mury_brilhante@hotmail.com \\ Maria de Fátima Duarte Marinho \\ ORCID: https://orcid.org/0000-0002-1321-0168 \\ Universidade Federal do Rio Grande do Norte, Brasil \\ E-mail: mariamarinhofisio@hotmail.com \\ Adriana Gomes Magalhães \\ ORCID: https://orcid.org/0000-0002-0279-5930 \\ Universidade Federal do Rio Grande do Norte, Brasil \\ E-mail: adriana fsm@yahoo.com.br \\ Grasiéla Nascimento Correia \\ ORCID: https://orcid.org/0000-0002-2722-5205 \\ Universidade Federal do Rio Grande do Norte, Brasil \\ E-mail: grasiela_n_correia@yahoo.com.br
}

\begin{abstract}
Resumo
Introdução:A pandemia de SARS-CoV-2 gerou um impacto na saúde sexual e reprodutiva principalmente por ser uma doença com alta transmissibilidade entre indivíduos que mantém contato próximo. Objetivo: Avaliar o impacto do isolamento social na função sexual, qualidade de vida, e autoimagem de mulheres nulíparas, antes e durante a pandemia de SARS-CoV-2. Métodos: Estudo analítico observacional longitudinal, com mulheres de 18 a 35 anos, nulíparas, com ensino médio completo, que não realizaram tratamento fisioterapêutico prévio. A coleta de dados contemplava dados antroposociodemográficos e os questionários Female Sexual Function Index (FSFI), Short Form Health Survey 36 (SF36), e a Escala de Silhuetas de Stunkard, Sorensen e Schlusinger (avaliação da autoimagem), aplicados antes e durante o isolamento social. A análise estatística foi realizada no GraphPad Prisma 6.0, e foi utilizado o teste de Shapiro Wilk, para analisar a distribuição da amostra, que foi não paramétrica, em seguida aplicou-se o Teste de Wilcoxon e a Correlação de Spearman. O nível de significância adotado foi $\mathrm{p} \leq 0,05$. Resultados: Foram avaliadas 37 voluntárias, as variáveis sociodemográficas e o índice de massa corporal não apresentaram diferença entre as avaliações, durante o isolamento social o número de mulheres sedentárias aumentou ( $\mathrm{p}<0.0001)$, a função orgástica melhorou $(\mathrm{p}=0,0081)$, a dor piorou significativamente no SF-36 (<0.0001), e quanto melhor foi o Estado Geral de Saúde do SF36 melhor foi a função sexual [p=0,04; $r=0,37]$, na correlação da atividade física, o tipo de isolamento social e o escore total obtido no questionário FSFI, não foi verificado correlações significativas. Conclusão: O isolamento social, favoreceu a melhora da função orgástica, a piora da dor no SF-36, e não interferiu na insatisfação da imagem corporal, que permaneceu semelhante.
\end{abstract}

Palavras-chave: Infecções por Coronavírus; Assoalho Pélvico; Disfunções Sexuais; Qualidade de Vida; Autoimagem.

\begin{abstract}
Introduction: The SARS-CoV-2 pandemic had an impact on sexual and reproductive health mainly because it is a disease with high transmissibility among individuals in close contact. Objective: Assess the impact of social isolation on sexual function, quality of life, and self-image of nulliparous women, before and during the SARS-CoV-2 pandemic. Methods: Longitudinal observational analytical study, with women aged 18 to 35 years, nulliparous, with complete high school education, who had not undergone previous physical therapy treatment. The data collection included anthroposociodemographic data and the Female Sexual Function Index (FSFI), Short Form Health Survey 36 (SF-36) questionnaires, and the Stunkard, Sorensen and Schlusinger Silhouettes Scale (self-image assessment), applied before and during social isolation. Statistical analysis was performed on GraphPad Prisma 6.0, and the Shapiro Wilk test was used to analyze the sample distribution, which was nonparametric, then the Wilcoxon test and Spearman's correlation
\end{abstract}


were applied. The level of significance adopted was $\mathrm{p} \leq 0.05$. Results: 37 volunteers were evaluated, the sociodemographic variables and the body mass index showed no difference between the evaluations, during social isolation the number of sedentary women increased $(\mathrm{p}<0.0001)$, the orgastic function improved $(\mathrm{p}=0.0081)$, the pain worsened significantly in SF-36 $(<0.0001)$, and the better the General Health Status of SF36, the better the sexual function $[\mathrm{p}=0.04 ; \mathrm{r}=0.37]$, in the correlation of physical activity, the type of social isolation and the total score obtained in the FSFI questionnaire, no significant correlations were found. Conclusion: Social isolation favored the improvement of orgastic function, the worsening of pain in the SF-36, and did not interfere with the dissatisfaction of body image, which remained similar.

Keywords: Coronavirus infections; Pelvic Floor; Sexual Dysfunctions; Quality of life; Self image.

\section{Resumen}

Introducción: La pandemia del SARS-CoV-2 tuvo un impacto en la salud sexual y reproductiva principalmente porque es una enfermedad con alta transmisibilidad entre personas en contacto cercano. Objetivo: Evaluar el impacto del aislamiento social en la función sexual, la calidad de vida y la autoimagen de las mujeres nulíparas, antes y durante la pandemia de SARS-CoV-2. Métodos: Estudio analítico observacional longitudinal, con mujeres de 18 a 35 años, nulíparas, con estudios secundarios completos, que no habían recibido tratamiento fisioterapéutico previo. La recolección de datos incluyó datos antroposociodemográficos y el Índice de Función Sexual Femenina (FSFI), los cuestionarios Short Form Health Survey 36 (SF-36) y la Escala de Siluetas de Stunkard, Sorensen y Schlusinger (evaluación de la autoimagen), aplicada antes y durante el aislamiento social. El análisis estadístico se realizó en GraphPad Prisma 6.0, y se utilizó la prueba de Shapiro Wilk para analizar la distribución de la muestra, la cual fue no paramétrica, luego se aplicó la prueba de Wilcoxon y la correlación de Spearman. El nivel de significancia adoptado fue $\mathrm{p} \leq 0.05$. Resultados: Se evaluaron 37 voluntarias, las variables sociodemográficas y el índice de masa corporal no mostraron diferencia entre las evaluaciones, durante el aislamiento social aumentó el número de mujeres sedentarias ( $\mathrm{p}$ $<0,0001)$, la función orgástica mejoró ( $\mathrm{p}=0,0081)$, el dolor empeoró significativamente en SF-36 $(<0,0001)$, y cuanto mejor es el estado de salud general de SF36, mejor es la función sexual [ $p=0,04 ; r=0,37$, en la correlación de la actividad física, el tipo de aislamiento social y la puntuación total obtenida en el cuestionario FSFI, no se encontraron correlaciones significativas. Conclusión: El aislamiento social favoreció la mejora de la función orgástica, el agravamiento del dolor en el SF-36, y no interfirió con la insatisfacción de la imagen corporal, que se mantuvo similar. Palabras clave: Infecciones por coronavirus; Piso pelvico; Disfunciones sexuales; Calidad de vida; Auto imagen.

\section{Introdução}

A pandemia de SARS-CoV-2 gerou um impacto na saúde sexual e reprodutiva de várias formas, tanto em nível individual, quanto social (Hussein, 2020), principalmente por ser uma doença com alta transmissibilidade entre indivíduos que mantém contato próximo (Koo, Ahn, Lim, Cho, \& Park, 2017). Devido a essa característica os governos de diversos países ordenaram o fechamento de espaços de reunião e a promoção do distanciamento social com o objetivo de evitar a disseminação da SARSCoV-2, o que culminou na mudança de alguns hábitos sexuais (Ibarra, Mehrad, Mauro, Godoy, Cruz, Nilforoushzadeh, \& Russo, 2020).

Do ponto de vista biofisiológico, os quadros de estresse, depressão e ansiedade provocados por esse contexto de incertezas geradas pela pandemia da SARS-CoV-2 (Maia \& Dias, 2020) associadas às dificuldades nos relacionamentos, advindos do convívio familiar 24 horas por dia, o isolamento social, as dificuldades financeiras e a incapacidade de gerar outras fontes de rendas, durante o período de quarentena favoreceram a piora do desempenho sexual (Hussein, 2020; Ribeiro \& Valle, 2020; Lai, Shih, Ko, Tang \& Hsueh, 2020), que entre as mulheres tem levado a queixas de: dispareunia, falta de excitação e falta de desejo sexual (Ribeiro \& Valle, 2020; Gonçalves, Gherman, Abdo, Nardi, \& Appolinário, 2020). Isso ocorre devido a resposta fisiológica associada ao estresse, ao medo e ao trauma, que promovem um aumento na síntese de cortisol, que em mulheres pode causar escores mais baixos nos domínios Excitação, Desejo e Satisfação no Índice de Função Sexual Feminina (Hamilton, Rellini \& Meston, 2008).

Alguns estudos têm sugerido mudança no comportamento sexual, pesquisas realizadas na China (Li, Li, Xin, Wang, \& Yang, 2020), Portugal (Carvalho \& Pascoal, 2020) e nos estados do Reino Unido (Jacob, et al., 2020) identificaram que tanto as atividades sexuais quanto a satisfação sexual de homens e mulheres jovens diminuíram durante a pandemia de SARS-CoV-2 (Li, Li, Xin, Wang, \& Yang, 2020; Carvalho \& Pascoal, 2020; Jacob, et al., 2020). 
Entretanto atualmente não foram encontrados estudos que avaliassem o impacto do isolamento social na função sexual de mulheres jovens e nulíparas, por isso, se faz necessária a realização de estudos que avaliem o impacto que o isolamento social pode causar na função sexual nesse público específico. Deste modo o objetivo primário do presente estudo foi avaliar o impacto do isolamento social em mulheres jovens e nulíparas residentes no interior do Estado do Rio Grande do Norte, devido a pandemia de SARS-CoV-2 sobre a função sexual, e como objetivo secundário pretende-se identificar a prevalência de disfunção sexual, avaliar a qualidade de vida, e a autoimagem das voluntárias antes e durante a pandemia causada pelo SARS-CoV-2, bem como correlacionar a qualidade de vida com a função sexual durante esse período.

\section{Métodos}

Trata-se de um estudo de caráter analítico observacional longitudinal, com recrutamento da amostra por demanda espontânea na Clínica Escola de Fisioterapia da Faculdade de Ciências da Saúde do Trairi (FACISA/UFRN), localizada no município de Santa Cruz no Estado do Rio Grande do Norte, no período de agosto de 2019 a setembro de 2020, sendo realizada a avaliação inicial 6 meses antes da pandemia de Sars-CoV-2 ser declarada pela OMS, e a segunda avaliação durante o isolamento social devido à pandemia.

Foram incluídas mulheres de 18 a 35 anos, nulíparas, com pelo menos o ensino médio completo, que não realizaram tratamento fisioterapêutico para IU, e que aceitaram participar da pesquisa. Foram excluídas aquelas que apresentaram relato de infecção uroginecológica, prolapso de órgãos pélvicos maior que grau II, desordens cognitivas e neurológicas que comprometessem a avaliação, hipertensão arterial descontrolada, terapia de reposição hormonal, alergia a látex e as que não conseguiram ser contactadas durante a pandemia. A pesquisa foi realizada de acordo com a resolução 466/2012, sendo aprovada pelo Comitê de Ética e Pesquisa da UFRN, sob o parecer número 4.247.124, e CAAE: 10000019.6.0000.5292.

A coleta de dados foi composta por uma anamnese, com informações pessoais referentes a idade, dados sociodemográficos, histórico ginecológico, obstétrico, urológico e prática de atividade física, coletados em uma ficha específica, a mesma continha os questionários que englobavam função sexual, qualidade de vida e autoimagem.

A função sexual, foi avaliada por meio do instrumento FSFI, com domínios multidimensionais, leva em consideração as respostas nas fases de desejo sexual, excitação sexual, lubrificação vaginal, orgasmo, satisfação sexual e dor (Rosen, 2002), utiliza dezenove perguntas referentes a função sexual nas últimas quatro semanas, e para cada pergunta existe um padrão de resposta, sendo atribuída a cada resposta uma pontuação de 0 a 5, onde 0 se refere a ausência de relação sexual no período referido, seu escore final é obtido pela soma de cada domínio, multiplicada por um fator de homogeneização (Rosen, 2000). A soma de cada pontuação de domínio individual é obtida e, em seguida, os valores de cada domínio recebem pesos diferentes para obter uma pontuação final máxima de 36 (Rosen, 2000). Esse instrumento foi validado para a população inglesa por Meston (2003) e já passou pelo processo de adaptação transcultural e validação para o português (Pacagnella, Martinez \& Vieira, 2009). O escore total pode variar de 2 a 36 pontos, com valores $\leq 26$ indicando disfunção sexual (Pacagnella, Martinez \& Vieira, 2009). Seus domínios também podem ser analisados de forma categorizada, conforme proposto por Jamali et al. (2016), onde valores menores que 4,28 para desejo, 5,08 para excitação, 5,45 para lubrificação, 5,05 para orgasmo 5,04 para satisfação e 5,51 para dor indicam a presença de disfunção nos referidos componentes (Jamali, Rahmanian, \& Javadpour, 2016).

Já para a avaliação da qualidade de vida, foi utilizado o SF-36, que possui 36 questões, englobando a capacidade funcional, aspectos físicos, dor, estado geral da saúde, vitalidade, aspectos sociais, aspectos emocionais e saúde mental, além de uma questão comparativa entre a saúde atual e a de um ano atrás saúde (Ware \& Sherbourne, 1992; Ware \& Gandek, 1994). Apresenta uma versão validada para o português, sua aplicação resulta em um valor específico para cada questão, que posteriormente levará a uma escala que representa desde uma menor qualidade de vida, quando obtém-se valores próximos de 0 , até uma melhor 
qualidade de vida, quando suas pontuações são mais próximas de 100, suas dimensões são avaliadas separadamente (Ciconelli, Ferraz, Santos, Meinão, \& Quaresma, 1999).

E a percepção da autoimagem, foi avaliada através da Escala de Silhuetas de Stunkard, Sorensen e Schlusinger (1983). Essa escala foi validade para mulheres brasileiras por Scagliusi (2006), ela representa por meio de figuras desde a magreza (silhueta 1) até a obesidade grave (silhueta 9), e a mulher avaliada deverá identificar qual figura representa melhor a sua silhueta atual e com qual ela gostaria de estar, a insatisfação será identificada quando a silhueta escolhida não for compatível com a imagem do corpo real, em relação ao seu IMC (Stunkard, Sorensen \& Schulsinger, 1983). A categorização para determinar essa satisfação foi: 0 satisfeitas; \pm 1 insatisfeitas; e \pm 2 ou mais muito insatisfeita.

Com relação ao isolamento social, elas foram alocadas em 3 tipos: Isolamento Total - Quando referiram não estar saindo de casa para realizar nenhuma atividade (sendo esse considerado o mais restrito); Isolamento Parcial: Quando as mesmas estavam saindo apenas para serviços essenciais, como farmácias e supermercados; não estava em isolamento: As que referiram ser profissional de saúde e precisou continuar trabalhando.

As variáveis coletadas no presente estudo foram classificadas em dois tipos, quantitativa e qualitativa. Inicialmente os dados foram tabulados no software Microsoft Excel®, e em seguida todas as análises estatísticas foram realizadas com o programa GraphPad Prisma®, versão 6.0, foram analisadas medidas de tendência central e dispersão, e posteriormente as inferenciais. Utilizou-se o Teste de Shapiro-Wilk para analisar a distribuição dos dados, identificou-se uma distribuição não normal, deste modo o teste de escolha para análise das variáveis não paramétricas foi o teste de Wilcoxon para a comparação dos resultados obtidos antes e durante o isolamento social devido a SARS-CoV-2. Para verificar o grau de correlação entre as variáveis, foi utilizado o Coeficiente de Correlação de Spearman, as correlações foram classificadas em forte ( $r>0,70)$, moderada $(r>0,40$ e $<0,69)$, fraca $(r<0,3)$ ou inexistente $(r<0,1)$ (Dancey \& Reidy, 2006). O nível de significância adotado foi de $p \leq 0,05$.

\section{Resultados}

A primeira avaliação contou com a participação de 40 voluntárias, que fizeram parte de um estudo realizado anteriormente, porém na segunda avaliação não foi possível entrar em contato com 3 voluntárias, por isso elas foram excluídas de ambas as coletas de dados. Então, 37 voluntárias realizaram as duas avaliações, como ilustrado na Figura 1. 
Figura 1 - Fluxograma de recrutamento e avaliação.

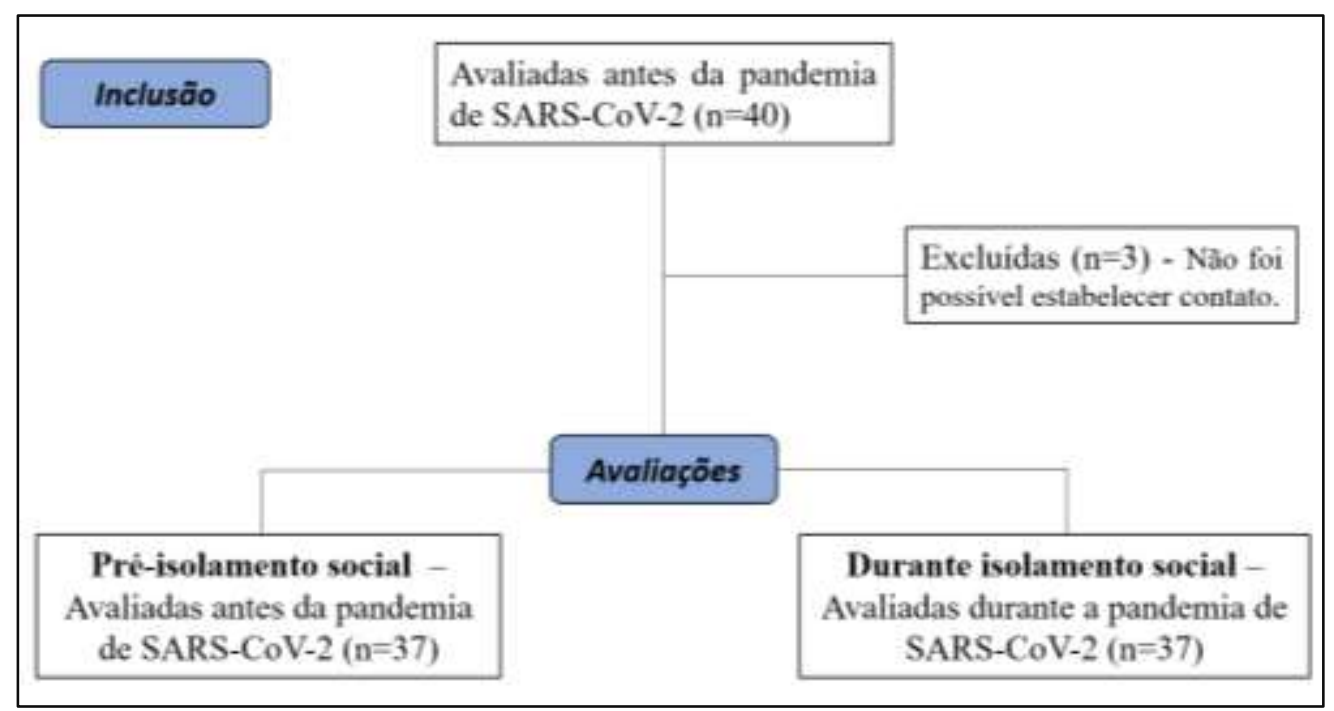

Fonte: Autores.

Os dados descritivos e as características das participantes estão apresentadas na Tabela 1. As variáveis sociodemográficas e IMC não apresentaram diferença entre as avaliações, demonstrando a homogeneidade da amostra. Houve uma diminuição no número de mulheres fisicamente ativas ( $\mathrm{p}<0.0001)$ e um aumento para 97,30\% das mulheres que estavam em isolamento parcial ou total durante a pandemia de SARS-CoV-2 ( $<<0.0001)$. Não foi verificada diferença em relação ao número de mulheres sexualmente ativas antes e durante o isolamento social.

Tabela 1 - Dados sociodemográficos e caracterização da amostra (n=37).

\begin{tabular}{|c|c|c|c|}
\hline & $\begin{array}{c}\text { Antes do isolamento social }(\mathbf{n}=37) \\
\text { Mediana [DIQ] } \\
\text { n (\%) }\end{array}$ & $\begin{array}{c}\text { Durante o isolamento social } \\
(\mathbf{n}=37) \\
\text { Mediana [DIQ] } \\
\text { n (\%) }\end{array}$ & p - Valor \\
\hline Idade (Anos) & $21,00[20,00-22,00]$ & $22,00[21,00-23,00]$ & 0,1893 \\
\hline $\operatorname{IMC}\left(\mathrm{Kg} / \mathbf{m}^{2}\right)$ & $23,60[21,35-25,25]$ & $23,50[22,05-25,20]$ & 0,6291 \\
\hline Renda (reais) & $2090,00[1045-2090]$ & $1045,00[1045-2090]$ & 0,2538 \\
\hline \multicolumn{4}{|l|}{ Nível Educacional } \\
\hline Ensino Médio Completo & $3(8,11 \%)$ & $3(8,11 \%)$ & \multirow{3}{*}{0,2500} \\
\hline Superior Incompleto & $30(81,08 \%)$ & $29(78,38 \%)$ & \\
\hline Superior Completo & $4(10,81 \%)$ & $5(13,51)$ & \\
\hline \multicolumn{4}{|l|}{ Estado civil } \\
\hline Casada & $1(2,71 \%)$ & $2(5,41 \%)$ & \multirow[t]{2}{*}{0,5000} \\
\hline Solteira & $36(97,29 \%)$ & $35(94,59 \%)$ & \\
\hline \multicolumn{4}{|l|}{ História Sexual } \\
\hline Sexualmente ativo & $33(89,18 \%)$ & $29(78,37 \%)$ & 0,5000 \\
\hline \multicolumn{4}{|l|}{ Isolamento Social } \\
\hline Total & $00(0,0 \%)$ & $06(16,22 \%)$ & \multirow{3}{*}{$<0,0001$} \\
\hline Parcial & $00(0,0 \%)$ & $30(81,08 \%)$ & \\
\hline Não está em isolamento & $37(100 \%)$ & $01(2,70 \%)$ & \\
\hline \multicolumn{4}{|l|}{ Atividade física } \\
\hline Sedentária & $13(35,14 \%)$ & $21(56,76 \%)$ & \multirow[t]{2}{*}{$<0,0001$} \\
\hline Ativa fisicamente & $24(64,86 \%)$ & $16(43,24 \%)$ & \\
\hline Caminhada & $01(04,17 \%)$ & $01(06,25 \%)$ & 0,0060 \\
\hline Corrida & $03(12,50 \%)$ & $04(25,00 \%)$ & $\mathbf{0 , 0 2 3 4}$ \\
\hline Musculação & $16(66,67 \%)$ & $08(50,00 \%)$ & $<0,0001$ \\
\hline Dança & $02(08,33 \%)$ & $01(06,25 \%)$ & $\mathbf{0 , 0 0 3 4}$ \\
\hline Cross Training & $02(08,33 \%)$ & $02(12,50 \%)$ & 0,0060 \\
\hline
\end{tabular}


Legenda: As variáveis numéricas estão descritas em mediana e DIQ: distância interquartílica, enquanto as variáveis categóricas são descritas frequência relativa e absoluta. IU: Incontinência Urinária. EF: Exercício Físico; Teste de Normalidade Shapiro-Wilk e Comparação das variáveis não paramétricas realizadas através do Teste de Wilcoxon. Fonte: Autores.

Em relação a função sexual avaliada pelo FSFI, houve diferença significativa apenas no domínio orgasmo (p=0,0081), demonstrando que a função orgástica das mulheres durante o isolamento social foi melhor, já os demais domínios e no escore total do FSFI não apresentou diferença significativa. E com relação ao SF-36, apenas o domínio dor piorou durante a isolamento social $(<0,0001)$, e na avaliação da autoimagem não foi verificada diferença estatística $(\mathrm{p}=0,1168)$.

Tabela 2. Comparação dos domínios dos questionários FSFI, SF-36 e a Escala de Silhuetas de Stunkard, Sorensen e schlusinger das mulheres sexualmente ativas de acordo com os grupos antes e durante a pandemia.

\begin{tabular}{lccc}
\hline & $\begin{array}{c}\text { Antes do isolamento social (n=33) } \\
\text { Mediana [DIQ] }\end{array}$ & $\begin{array}{c}\text { Durante o isolamento social } \\
(\mathbf{n = 2 9}) \\
\text { Mediana [DIQ] }\end{array}$ & P - Valor \\
\hline FSFI & $27,10[20,90-30,35]$ & $27,70[22,65-31,15]$ & 0,2897 \\
$\quad$ Pontuação Total & $04,02[03,60-04,80]$ & $03,60[03,00-04,50]$ & 0,3782 \\
Domínio Desejo & $04,80[31,50-05,40]$ & $04,50[03,75-05,40]$ & 0,4998 \\
Domínio Excitação & $04,80[02,55-05,40]$ & $05,70[03,45-06,60]$ & 0,0733 \\
Domínio Lubrificação & $04,00[01,40-04.80]$ & $05,20[03,60-05,80]$ & $\mathbf{0 , 0 0 8 1}$ \\
Domínio Orgasmo & $05,20[03,80-05,60]$ & $05,60[03,60-05,60]$ & 0,6281 \\
Domínio Satisfação & $04,40[03,20-06,60]$ & $03,60[03,20-04,80]$ & 0,4688 \\
Domínio Dor & & & \\
\hline SF-36 & $90,00[80,00-97,50]$ & $90,00[80,00-95,00]$ & 0,6909 \\
Capacidade Funcional & $75,00[75,00-100,0]$ & $100,0[50,00-100,0]$ & 0,6580 \\
Limitação Física & $51,00[32,50-67,00]$ & $22,00[11,00-34,00]$ & $<\mathbf{0 , 0 0 0 1}$ \\
Dor & $42,00[32,00-71,00]$ & $62,00[41,00-71,00]$ & 0,3742 \\
Estado Geral de Saúde & $45,00[27,50-55,00]$ & $45,00[25,00-55,00]$ & 1,0000 \\
Vitalidade & $75,00[50,00-87,50]$ & $75,00[50,00-81,25]$ & 0,9321 \\
Aspectos Sociais & $33,30[00,00-66,70]$ & $00,00[00,00-66,70]$ & 0,6609 \\
Aspectos Emocionais & $56,00[42,00-72,00]$ & $60,00[46,00-68,00]$ & 0,5331 \\
Saúde Mental & $1,00[0,00-1,00]$ & $1,00[0,00-2,00]$ & 0,1168 \\
\hline Autoimagem & & & \\
\hline
\end{tabular}

Legenda: As variáveis numéricas estão descritas em mediana e DIQ: distância interquartílica, enquanto as variáveis categóricas são descritas frequência relativa e absoluta. Teste de Normalidade Shapiro-Wilk e Comparação das variáveis não paramétricas realizadas através do Teste de Wilcoxon. * $\mathrm{p}<0.05$ diferença estatisticamente significante. Fonte: Autores.

Na Tabela 3 é apresentada a correlação entre os domínios do SF-36 e o escore total do FSFI das mulheres ativas sexualmente. Apenas o domínio Estado Geral de Saúde do SF36 apresentou correlação [p=0,04; r=0,37], sendo ela classificada como uma correlação positiva fraca, ou seja, quanto melhor a função sexual das mulheres avaliadas, melhor o estado geral de saúde. 
Tabela 3 - Correlações entre os domínios do SF-36 e o escore total obtido no FSFI das mulheres ativas sexualmente.

\begin{tabular}{l|ccc|c}
\hline \multirow{2}{*}{\multicolumn{1}{c}{ Variáveis }} & \multicolumn{2}{c|}{ Antes do isolamento social (n=33) } & \multicolumn{2}{c}{ Durante o isolamento social (n=29) } \\
\cline { 2 - 5 } & $\mathbf{p}$ & $\mathbf{r}$ & $\mathbf{p}$ & $\mathbf{r}$ \\
\hline Capacidade Funcional & 0,212 & 0,226 & 0,594 & $-0,103$ \\
Limitação Física & 0,487 & $-0,127$ & 0,383 & 0,168 \\
Dor & 0,466 & 0,133 & 0,858 & 0,034 \\
Estado Geral de Saúde & 0,996 & 0,001 & $\mathbf{0 , 0 4 2}$ & 0,378 \\
Vitalidade & 0,606 & $-0,094$ & 0,545 & 0,116 \\
Aspectos Sociais & 0,454 & $-0,137$ & 0,997 & $-0,001$ \\
Aspectos Emocionais & 0,769 & $-0,053$ & 0,717 & $-0,070$ \\
Saúde Mental & 0,774 & $-0,052$ & 0,098 & 0,313 \\
\hline
\end{tabular}

Legenda: SF-36: Medical Outcomes Short-Form Health Survey; FSFI: Female Sexual Function Index; PDFI-20: Instrumentos de Inabilidade do Assoalho Pélvico; PFIQ-7: Questionário de Impacto no Assoalho Pélvico; r Coeficiente de Correlação de Spearman. Fonte: Autores.

$\mathrm{Na}$ correlação da atividade física, o tipo de isolamento social e o escore total obtido no questionário FSFI, não foi verificado correlações significativas.

Tabela 4 - Correlações entre a atividade física, o isolamento social total, parcial ou nenhum e a pontuação total no FSFI.

\begin{tabular}{ccccccc}
\hline & \multicolumn{2}{c}{ Isolamento Social $(\mathbf{n = 2 9})$} & \multicolumn{3}{c}{ Atividade Física $(\mathbf{n}=\mathbf{2 9})$} \\
\cline { 2 - 7 } & $\mathbf{p}$ & $\mathbf{R}$ & $\mathbf{p}$ & \multicolumn{3}{c}{ Durante } \\
& 0,875 & $-0,030$ & 0,476 & $-0,128$ & 0,843 & 0,038 \\
\hline FSFI & & & & $\mathbf{p}$ & $\mathbf{p}$ \\
\hline
\end{tabular}

Legenda: FSFI: Female Sexual Function Index; PDFI-20: Instrumentos de Inabilidade do Assoalho Pélvico; PFIQ-7: Questionário de Impacto no Assoalho Pélvico; r - Coeficiente de Correlação de Spearman. Fonte: Autores.

\section{Discussão}

O presente estudo teve como objetivo avaliar o impacto do isolamento social devido a pandemia de SARS-CoV-2 na função sexual, e identificar a prevalência de disfunção sexual, o impacto do isolamento sobre a qualidade de vida, e a autoimagem das participantes antes e durante a pandemia causada pelo SARS-CoV-2, bem como correlacionar a qualidade de vida com a função sexual de antes e durante isolamento social.

O impacto da pandemia da SARS-CoV-2, em seus diferentes aspectos, na função sexual durante o isolamento social já vem sendo estudo em países como China ( $\mathrm{Li}, \mathrm{Li}$, Xin, Wang, \& Yang, 2020), Portugal (Carvalho \& Pascoal, 2020), Reino Unido (Jacob, et al., 2020) Itália (Schiavi, Spina, Zullo, Colagiovanni, Luffarelli, Rago, \& Palazzetti, 2020) e Gana (Sefakor Ametepe, Okere, \& Anto-Ocrah, 2020), apresentando por sua vez diferentes resultados. No Brasil, não foram encontrados estudos que tivessem os objetivos propostos no presente trabalho, até o momento as pesquisas desenvolvidas são voltadas para outras temáticas como combate a COVID-19, violência doméstica, saúde sexual, reprodução e fertilidade (Coutinho, Lima, Leocádio, $\&$ Bernardes, 2020). 
Em nosso estudo realizado com mulheres jovens, solteiras, nulíparas, residentes no interior do estado do Rio Grande do Norte, foi observado que o número de participantes em isolamento social aumentou significativamente, pois antes da SARSCOV-2 não havia a necessidade de isolamento social, fato este que já era esperado pelos autores deste estudo. Em contrapartida o nível de atividade física teve uma diminuição, e a vida sexual ativa nas 4 semanas anteriores a segunda avaliação apresentou uma pequena redução, mas sem diferença estatística, de 89,18\% antes do isolamento social, para 78,37\% durante o isolamento, sendo esse resultado convergente com o que foi apontado em outros estudos (Li, Li, Xin, Wang, \& Yang, 2020; Carvalho \& Pascoal, 2020; Jacob, et al., 2020). Já Fuchs et al (2020) encontraram resultados diferentes, cujo aumento da prevalência de disfunção sexual entre as mulheres avaliadas antes e durante a pandemia foi estatisticamente significante, saindo de $15,3 \%$ para 34,3\% (Fuchs, Matonóg, Pilarska, Sieradzka, Szul, Czuba, \& Drosdzol-Cop, 2020).

Jacob et al. (2020) verificaram que 60,1\% da amostra estudada relatou não ser sexualmente ativa durante o isolamento social. Entretanto no presente estudo, apenas $21,63 \%$ das voluntárias não estavam ativas sexualmente durante o isolamento social. Um ponto importante, é que a amostra no presente estudo foi composta por 94,59\% das participantes solteiras, grande maioria em isoladas social e provavelmente estavam distante de seus parceiros, e mesmo assim o número de mulheres sexualmente ativas, não sofreu uma redução. Essa atividade sexual pode ter ocorrido por meio do contato físico, virtual e por automasturbação, entretanto não foi objeto do estudo a discriminação do tipo de atividade sexual das voluntárias.

$\mathrm{O}$ isolamento social devido a SARS-CoV-2 favoreceu as mudanças na forma de procurar e viver relações amorosas e sexuais, onde paqueras, afetos, amores e prazeres sexuais, que nas sociedades conectadas já aconteciam num hibridismo de encontros físicos e virtuais, em sites e aplicativos, com o avanço da pandemia passaram, para aqueles que vivem em isolamento físico, a ser reinventados apenas nos domínios das redes digitais (da Silva, da Silva Junior, \& Couto, 2020). Tecchio (2020) ressalta que, com a pandemia, as pessoas têm procurado novas formas de se conectar, notado principalmente pelo aumento em $37 \%$ nos acessos a aplicativos de namoro (TECCHIO et al, 2020). O isolamento físico tem afetado o estado emocional das pessoas, muitos procuram nos aplicativos de paquera novas funções como romance, vínculos afetivos mais estáveis e duradouros, relacionamentos mais sensíveis e profundos e, também, sexo casual virtual (da Silva, da Silva Junior, \& Couto, 2020). O mundo virtual expressa e excita jogos sexuais em meio às histórias e reinvenções de si nas vertigens do gozo (LIMA et al, 2020). Por seu intermédio, aprendemos sobre novas formas de experimentar amor, relacionamentos, sexo e afeto, a partir de distanciamentos físicos e de interações mediadas por tecnologias com pessoas que podem estar em qualquer lugar do mundo (da Silva, da Silva Junior, \& Couto, 2020).

Molllaioli et al (2020) verificou que metade de sua amostra (50,3\%) relatou interrupção da atividade sexual durante o isolamento social, por duas razões possíveis: sofrimento devido à quarentena e impossibilidade de ter o parceiro sexual ao seu alcance (Mollaioli, Sansone, Ciocca, Limoncin, Colonnello, Di Lorenzo, \& Jannini, 2020). Além disso, Fuchs et al (2020) aponta que a maioria das mulheres declararam que a redução da atividade sexual se deu por diferentes motivos, entre eles, 41,5\% falaram do isolamento do companheiro, 39,3\% sentem falta de desejo devido ao estresse, $16 \%$ apontam como causa desentendimentos com o companheiro e apenas 3,2\% das mulheres temiam que o SARS-CoV-2 pudesse ser transmitido durante o contato sexual (Fuchs, Matonóg, Pilarska, Sieradzka, Szul, Czuba, \& Drosdzol-Cop, 2020). Entretanto, apesar das mulheres avaliadas nesse estudo terem uma média de idade semelhante a do presente estudo, 92,8\% de sua amostra eram de mulheres casadas ou que estavam em um relacionamento informal, e 27,6\% delas tinham um ou mais filhos, esses fatos por sua vez diferem da nossas amostras, tendo em vista que foram avaliadas apenas mulheres nulíparas, que em sua maioria não tinham companheiros, o que pode ter favorecido esses resultados divergentes.

Essa interrupção da atividade sexual durante o período de pandemia de SARS-CoV-2, pode ser atribuída também as recomendações relacionadas a atividade sexual, que foram dadas por especialistas que se reuniram e publicaram um estudo 
(2020), orientando que sob nenhuma circunstância o sexo deve ser realizado com um novo parceiro, a menos que haja a certeza de que o parceiro foi imunizado contra o vírus, para quem não tem companheiro e casais que mantêm convivência separada, a recomendação é a abstinência de relações sexuais (Cabello, Sánchez, Farré, \& Montejo, 2020). Tendo em vista que a relação sexual requer contato físico próximo, e o SARS-CoV-2 é muito facilmente transmitido com esse nível de proximidade (Santarpia, et al., 2020).

De acordo com Fuchs et al (2020) e Yuksel \& Ozgor (2020) a pandemia provocou uma deterioração da qualidade de vida sexual entre as mulheres (Fuchs, Matonóg, Pilarska, Sieradzka, Szul, Czuba, \& Drosdzol-Cop, 2020; Yuksel \& Ozgor, 2020), em contrapartida o desejo sexual e a frequência das relações sexuais aumentaram durante o isolamento social (Yuksel \& Ozgor, 2020), já em nosso estudo, o que apresentou um aumento significativo durante o isolamento social foi a função orgástica, o que não corresponde aos resultados averiguados em outros estudos, tendo em vista que de acordo com eles, quanto mais prolongado o período em quarentena maior é o sofrimento psicológico, incluindo mau humor, ansiedade, depressão, irritabilidade e medo, podendo comprometer a apresentação da disfunção sexual (Brooks, Webster, Smith, Woodland, Wessely, Greenberg, \& Rubin, 2020).

Estudos epidemiológicos robustos evidenciam unanimamente que a presença e a gravidade da grande maioria das disfunções sexuais estão diretamente relacionadas à idade em ambos os sexos (Laumann, Nicolosi, Glasser, Paik, Gingell, \& Wang, 2005; Corona, et al., 2010; Hayes \& Dennerstein, 2005). Nosso estudo foi realizado com mulheres jovens, com idade entre 18 e 35 anos, nulíparas, período no qual as disfunções sexuais costumam ser menos prevalentes, pois de acordo com os estudos epidemiológicos, o aumento da idade parece ser um fator de risco para as disfunções sexuais (Laumann, Paik, \& Rosen, 1999; Rosen, Fisher, Eardley, Niederberger, \& Sand, 2004).

Em relação ao SF-36, apenas o domínio dor apresentou uma piora $(<0.0001)$ durante o isolamento social, fato este que não é abordado em outros estudos presentes na literatura, acreditamos que esse resultado pode ser atribuído ao nível de trabalhos domésticos, que podem influenciar a sobrecarga mecânica (Rosa, Zanini, Zimermmam, Ghisi, Policarpo, Dagostin, \& Salvador, 2020). Zomalheto et al. (2020) identificou em seu estudo que a deterioração da QV do paciente durante o período de confinamento esteve ligada a fatores como o medo de contrair a infecção por SARS-CoV-2, (p=0,001), medo de estigmatização $(p=0,009)$ e do desemprego temporário relacionado à infecção $(p=0,001)$, além de queda na renda mensal $(p=0,002)$, como se sabe, a dor é determinada de forma subjetiva, podendo ser influenciada por fatores psicossociais (Marquez, 2011).

Em nosso estudo, verificamos que quanto melhor a função sexual das mulheres avaliadas pelo FSFI, melhor foi o domínio estado geral de saúde no SF-36. A pandemia de SARS-CoV-2 afeta indiretamente a função sexual com implicações na saúde geral (Pennanen-Iire, et al., 2021), há indícios de que a atividade sexual é um contribuinte integral para a QV e saúde física geral, no qual os participantes que apresentam maior satisfação sexual relatam uma condição de saúde excelente (Flynn, et al., 2016). Em outro estudo realizado antes da pandemia de SARS-CoV-2, foi visto que a avaliação da função sexual feminina se fez importante, pois interferiu na QV e esteve associada a problemas gerais de saúde (Abdo, \& Fleury, 2006), corroborando com os nossos achados.

$\mathrm{Na}$ avaliação da autoimagem não houve diferença durante o isolamento social e não houve correlação com a função sexual, em contrapartida, outros estudos evidenciaram a existência de uma correlação direta entre a imagem corporal geral e as subescalas da função sexual, incluindo excitação sexual, desejo sexual, lubrificação, orgasmo, satisfação sexual e dispareunia (Kogure, et al., 2019).

Geralmente a função social e conjugal, os relacionamentos interpessoais e a QV de um indivíduo são afetadas pela imagem corporal negativa (Simon, et al., 2008), a preocupação das pessoas, principalmente das mulheres, sobre como os outros podem avaliar seu corpo afetam significativamente sua atividade sexual e QV (Asgari, \& Roshanak, 2010), estando a valorização da 
função sexual reduzida nas mulheres insatisfeitas com a forma corporal (Chang, Yang, \& Chen, 2019), porém esse resultado não foi encontrado em nosso estudo, apesar das participantes estarem insatisfeitas com a sua imagem corporal, elas eram eutróficas, e essa insatisfação não teve implicação na função sexual e qualidade de vida.

\section{Conclusão}

Diante dos achados encontrados no presente estudo, é possível concluir que o isolamento social proposto para controlar a disseminação da SARS-CoV-2, favoreceu a uma melhora da função orgástica, e não afetou os demais domínios do FSFI, e que apesar da prevalência da disfunção sexual ter aumentado de $42,42 \%$ para $44,82 \%$, esse aumento não foi estatisticamente significante em mulheres jovens e nulíparas. Com relação a QV avaliada pelo SF-36, o domínio dor apresentou uma piora durante o isolamento social e quanto melhor a função sexual melhor foi o estado geral de saúde avaliado pelo SF36. Já com relação a autoimagem, as voluntárias deste estudo permaneceram igualmente insatisfeitas com a sua imagem corporal.

\section{Considerações finais}

Embora os resultados deste estudo forneçam resultados preliminares acerca da função sexual, qualidade vida, e autoimagem de mulheres jovens, nulíparas, em isolamento social devido a SARS-CoV-2, é necessário ter cautela na generalização dos resultados, visto que esse foi um estudo pioneiro, realizado durante a pandemia temos como limitação uma amostra pequena, intencional e não aleatória, que utilizou um instrumento de qualidade de vida geral, e cujo questionário de autoimagem também é limitado, pois a presença de algum distúrbio de imagem como anorexia, bulimia e afins poderia enviesar os resultados.

Desse modo se faz necessário a realização de estudos com maior número amostral, que avaliem o tipo de atividade sexual que as mulheres estão realizando durante o isolamento social, especificando se foi por meio de contato físico, virtual ou automasturbação. Também são necessários estudos utilizando questionários de qualidade de vida específicos para função sexual, e que levem em consideração a forma como os distúrbios de imagem corporal poderiam ser minimizados, para que os resultados encontrados possam ser extrapolados a uma maior população de mulheres.

\section{Referências}

Abdo, C. H. N., \& Fleury, H. J. (2006). Aspectos diagnósticos e terapêuticos das disfunções sexuais femininas. Archives of Clinical Psychiatry (São Paulo), 33(3), 162-167. Doi: https://doi.org/10.1590/S0101-60832006000300006

Abdo, C. H. N., Oliveira, W. M., Moreira, E. D., \& Fittipaldi, J. A. S. (2004). Prevalence of sexual dysfunctions and correlated conditions in a sample of Brazilian women-results of the Brazilian study on sexual behavior (BSSB). International Journal of Impotence Research, 16(2), 160-166. Doi: https://doi.org/10.1038/sj.ijir.3901198

Asgari, P., \& Roshanak, S. (2010). A comparison between the quality of life, life satisfaction, sex role attitude \& self-esteem among students with high and low body image. Journal of thought \& behavior in clinical psychology, 5(17), 9-18. https://www.sid.ir/en/journal/ViewPaper.aspx?id=200286

Bedford, J., Enria, D., Giesecke, J., Heymann, D. L., Ihekweazu, C., Kobinger, G., ... \& Wieler, L. H. (2020). COVID-19: towards controlling of a pandemic. The lancet, 395(10229), 1015-1018. https://doi.org/10.1016/S0140-6736(20)30673-5

Brooks, S. K., Webster, R. K., Smith, L. E., Woodland, L., Wessely, S., Greenberg, N., \& Rubin, G. J. (2020). The psychological impact of quarantine and how to reduce it: rapid review of the evidence. The lancet, 395(10227), 912-920. https://doi.org/10.1016/S0140-6736(20)30460-8

Cabello, F., Sánchez, F., Farré, J. M., \& Montejo, A. L. (2020). Consensus on recommendations for safe sexual activity during the COVID-19 coronavirus pandemic. Journal of clinical medicine, 9(7), 2297. https://doi.org/10.3390/jcm9072297

Carvalho, J., \& Pascoal, P. M. (2020). Challenges in the Practice of Sexual Medicine in the Time of COVID-19 in Portugal. The journal of sexual medicine, 17(7), 1212-1215. https://doi.org/10.1016/j.jsxm.2020.05.024

Chang, S. R., Yang, C. F., \& Chen, K. H. (2019). Relationships between body image, sexual dysfunction, and health-related quality of life among middle-aged women: A cross-sectional study. Maturitas, 126, 45-50. https://doi.org/10.1016/j.maturitas.2019.04.218 
Ciconelli, R. M., Ferraz, M. B., Santos, W., Meinão, I., \& Quaresma, M. R. (1999). Tradução para a língua portuguesa e validação do questionário genérico de avaliação de qualidade de vida SF-36 (Brasil SF-36). Rev bras reumatol, 39(3), 143-50. https://www.researchgate.net/profile/WiltonSantos/publication/279904628_Brazilian-Portuguese_version_of_the_SF-

36_A_reliable_and_valid_quality_of_life_outcome_measure/links/5c6a236d4585156b57030388/Brazilian-Portuguese-version-of-the-SF-36-A-reliable-andvalid-quality-of-life-outcome-measure.pdf

Corona, G., Lee, D. M., Forti, G., O'Connor, D. B., Maggi, M., O'Neill, T. W., ... \& EMAS Study Group. (2010). Age-related changes in general and sexual health in middle-aged and older men: results from the European Male Ageing Study (EMAS). The journal of sexual medicine,7(4), 1362-1380. https://doi.org/10.1111/j.1743-6109.2009.01601.x

Coutinho, R. Z., Lima, L. C. D., Leocádio, V. A., \& Bernardes, T. (2020). Considerações sobre a pandemia de Covid-19 e seus efeitos sobre a fecundidade e a saúde sexual e reprodutiva das brasileiras. Revista Brasileira de Estudos de População, 37. https://doi.org/10.20947/s0102-3098a0130

Dancey C.P., \& Reidy J. Estatística sem matemática para psicologia: usando SPSS para Windows. 3rd ed. Artmed; 2006. 1-608.

da Silva, J. F., da Silva Junior, A. O., \& Couto, E. S. (2020). Amor, sexo e distância física. Revista Educação Em Questão, 58(58). https://doi.org/10.21680/19811802.2020v58n58ID21741

Flynn, K. E., Lin, L., Bruner, D. W., Cyranowski, J. M., Hahn, E. A., Jeffery, D. D., ... \& Weinfurt, K. P. (2016). Sexual satisfaction and the importance of sexual health to quality of life throughout the life course of US adults. The journal of sexual medicine, 13(11), 1642-1650. https://doi.org/10.1016/j.jsxm.2016.08.011

Fuchs, A., Matonóg, A., Pilarska, J., Sieradzka, P., Szul, M., Czuba, B., \& Drosdzol-Cop, A. (2020). The impact of COVID- 19 on female sexual health. International Journal of Environmental Research and Public Health, 17(19), 7152. https://doi.org/10.3390/ijerph17197152

Gonçalves, W. D. S., Gherman, B. R., Abdo, C. H. N., Nardi, A. E., \& Appolinário, J. C. B. (2019). Função e disfunção sexual na depressão: uma revisão sistemática. Jornal Brasileiro de Psiquiatria, 68(2), 110-120. https://doi.org/10.1590/0047-2085000000234

Hamilton, L. D., Rellini, A. H., \& Meston, C. M. (2008). Cortisol, sexual arousal, and affect in response to sexual stimuli. The journal of sexual medicine, 5(9), 2111-2118. https://doi.org/10.1111/j.1743-6109.2008.00922.x

Hayes, R., \& Dennerstein, L. (2005). The impact of aging on sexual function and sexual dysfunction in women: A review of population-based studies. The journal of sexual medicine, 2(3), 317-330. https://doi.org/10.1111/j.1743-6109.2005.20356.x

Hussein, J. (2020). COVID-19: What implications for sexual and reproductive health and rights globally? https://doi.org/10.1080/26410397.2020.1746065

Ibarra, F. P., Mehrad, M., Mauro, M. D., Godoy, M. F. P., Cruz, E. G., Nilforoushzadeh, M. A., \& Russo, G. I. (2020). Impact of the COVID-19 pandemic on the sexual behavior of the population. The vision of the east and the west. International braz $j$ urol, 46, 104-112. https://doi.org/10.1590/s16775538.ibju.2020.s116

Ibine, B., Sefakor Ametepe, L., Okere, M., \& Anto-Ocrah, M. (2020). "I did not know it was a medical condition": Predictors, severity and help seeking behaviors of women with female sexual dysfunction in the Volta region of Ghana. PloS one, 15(1), e0226404. https://doi.org/10.1371/journal.pone.0226404

Jacob, L., Smith, L., Butler, L., Barnett, Y., Grabovac, I., McDermott, D., ... \& Tully, M. A. (2020). Challenges in the practice of sexual medicine in the time of COVID-19 in the United Kingdom. The journal of sexual medicine, 17(7), 1229-1236. https://doi.org/10.1016/j.jsxm.2020.05.001

Jamali, S., Rahmanian, A., \& Javadpour, S. (2016). Examining the sexual function and related attitudes among aged women: A cross-sectional study. International Journal of Reproductive BioMedicine, 14(1), 29. https://www.ncbi.nlm.nih.gov/pmc/articles/PMC4837921/

Kogure, G. S., Ribeiro, V. B., Lopes, I. P., Furtado, C. L. M., Kodato, S., de Sá, M. F. S., ... \& Dos Reis, R. M. (2019). Body image and its relationships with sexual functioning, anxiety, and depression in women with polycystic ovary syndrome. Journal of affective disorders, 253, 385-393. https://doi.org/10.1016/j.jad.2019.05.006

Koo, S., Ahn, Y., Lim, J. Y., Cho, J., \& Park, H. Y. (2017). Obesity associates with vasomotor symptoms in postmenopause but with physical symptoms in perimenopause: a cross-sectional study. BMC women's health, 17(1), 1-8. https://bmcwomenshealth.biomedcentral.com/articles/10.1186/s12905-017-0487-7

Lai, C. C., Shih, T. P., Ko, W. C., Tang, H. J., \& Hsueh, P. R. (2020). Severe acute respiratory syndrome coronavirus 2 (SARS-CoV-2) and coronavirus disease2019 (COVID-19): The epidemic and the challenges. International journal of antimicrobial agents, 55(3), 105924. https://doi.org/10.1016/j.ijantimicag.2020.105924

Laumann, E. O., Nicolosi, A., Glasser, D. B., Paik, A., Gingell, C., Moreira, E., \& Wang, T. (2005). Sexual problems among women and men aged 40-80 y: prevalence and correlates identified in the Global Study of Sexual Attitudes and Behaviors. International journal of impotence research, 17(1), 39-57. https://doi.org/10.1038/sj.ijir.3901250

Laumann, E. O., Paik, A., \& Rosen, R. C. (1999). Sexual dysfunction in the United States: prevalence and predictors. Jama, 281(6), 537-544. https://doi.org/10.1001/jama.281.6.537

Li, W., Li, G., Xin, C., Wang, Y., \& Yang, S. (2020). Changes in sexual behaviors of young women and men during the coronavirus disease 2019 outbreak: a convenience sample from the epidemic area. The Journal of Sexual Medicine. https://www.ncbi.nlm.nih.gov/pmc/articles/PMC7188657/\#!po=87.5000

Lima, D. M., Couto, E. S., \& Silva, P. (2020). Manda nudes: Pedagogias sexuais no Grindr. ARTEFACTUM-Revista de estudos em Linguagens e Tecnologia, 19(1), 1-13. http://artefactum.rafrom.com.br/index.php/artefactum/article/view/1843

Maia, B. R., \& Dias, P. C. (2020). Anxiety, depression and stress in university students: the impact of COVID-19. Estudos de Psicologia (Campinas), 37. https://doi.org/10.1590/1982-0275202037e200067

Marquez, J. O. (2011). A dor e os seus aspectos multidimensionais. Ciência e Cultura, 63(2), 28-32. http://dx.doi.org/10.21800/S0009-67252011000200010 
Mollaioli, D., Sansone, A., Ciocca, G., Limoncin, E., Colonnello, E., Di Lorenzo, G., \& Jannini, E. A. (2021). Benefits of sexual activity on psychological, relational, and sexual health during the COVID-19 breakout. The journal of sexual medicine, 18(1), 35-49. https://doi.org/10.1016/j.jsxm.2020.10.008

Pacagnella, R. D. C., Vieira, E. M., Rodrigues Jr, O. M., \& Souza, C. D. (2008). Adaptação transcultural do female sexual function index. Cadernos de Saúde Pública, 24, 416-426. https://www.scielosp.org/article/csp/2008.v24n2/416-426/pt/

Pennanen-Iire, C., Prereira-Lourenço, M., Padoa, A., Ribeirinho, A., Samico, A., Gressler, M., ... \& Girard, A. (2021). Sexual health implications of COVID-19 pandemic. Sexual medicine reviews, 9(1), 3-14. https://doi.org/10.1016/j.sxmr.2020.10.004

Ribeiro, J. N., \& Valle, P. A. S. S. (2016). Disfunção Sexual Feminina. Revista Brasileira de Sexualidade Humana,27(2). https://doi.org/10.35919/rbsh.v27i2.109

Rosa, L., Zanini, M. T. B., Zimermmam, K. C. G., Ghisi, M. G., Policarpo, C. M., Dagostin, V. S., \& Salvador, M. B. (2017). Impacto no cotidiano de mulheres com incontinência urinária. Estima-Brazilian Journal of Enterostomal Therapy, 15(3). https://doi.org/10.5327/Z1806-3144201700030003

Rosen, R. C., Fisher, W. A., Eardley, I., Niederberger, C., Nadel, A., \& Sand, M. (2004). The multinational Men's Attitudes to Life Events and Sexuality (MALES) study: I. Prevalence oSf erectile dysfunction and related health concerns in the general population. Current medical research and opinion, 20(5), 607617. https://doi.org/10.1185/030079904125003467

Santarpia, J. L., Rivera, D. N., Herrera, V., Morwitzer, M. J., Creager, H., Santarpia, G. W., ... \& Lowe, J. J. (2020). Transmission potential of SARS-CoV-2 in viral shedding observed at the University of Nebraska Medical Center. MedRxiv. https://doi.org/10.1101/2020.03.23.20039446

Scagliusi, F. B., Alvarenga, M., Polacow, V. O., Cordás, T. A., de Oliveira Queiroz, G. K., Coelho, D., ... \& Lancha Jr, A. H. (2006). Concurrent and discriminant validity of the Stunkard's figure rating scale adapted into Portuguese. Appetite, 47(1), 77-82. https://doi.org/10.1016/j.appet.2006.02.010

Schiavi, M. C., Spina, V., Zullo, M. A., Colagiovanni, V., Luffarelli, P., Rago, R., \& Palazzetti, P. (2020). Love in the time of COVID-19: sexual function and quality of life analysis during the social distancing measures in a group of Italian reproductive-age women. The Journal of Sexual Medicine, 17(8), 1407-1413. https://doi.org/10.1016/j.jsxm.2020.06.006

Simon, G. E., Ludman, E. J., Linde, J. A., Operskalski, B. H., Ichikawa, L., Rohde, P., ... \& Jeffery, R. W. (2008). Association between obesity and depression in middle-aged women. General hospital psychiatry, 30(1), 32-39. https://doi.org/10.1016/j.genhosppsych.2007.09.001

Stunkard, A., Sorensen, T., \& Schlusinger, F. (1983). Use of Danish adoption register for the study of obesity and thinness 60(1), 115-120. https://www.scopus.com/record/display.uri?eid=2-s2.0-0020667098\&origin=inward\&txGid=7c0b299e53f8fe76f0d228ad2223570f

Tecchio, Manuela. (2020, May 13, 2020). Quarentena movimentada: apps como Tinder e Happn têm aumento de acessos. CNN Brasil Business, Rio de Janeiro, 13 maio 2020. https://www.cnnbrasil.com.br/business/2020/05/13/durante-isolamento-apps-como-tinder-e--happn-registram-aumento-nas-interacoes . Acesso em: 10 de março de 2021.

Ware Jr, J. E., \& Sherbourne, C. D. (1992). The MOS 36-item short-form health survey (SF-36): I. Conceptual framework and item selection. Medical care, 473-483. https://www.jstor.org/stable/3765916

Ware, Jr, J. E., \& Gandek, B. (1994). The SF-36 Health Survey: Development and use in mental health research and the IQOLA Project. International Journal of Mental Health, 23(2), 49-73. https://doi.org/10.1080/00207411.1994.11449283

Wiegel, M., Meston, C., \& Rosen, R. (2005). The female sexual function index (FSFI): cross-validation and development of clinical cutoff scores. Journal of sex \& marital therapy, 31(1), 1-20. https://doi.org/10.1080/00926230590475206.

Wu, J. T., Leung, K., \& Leung, G. M. (2020). Nowcasting and forecasting the potential domestic and international spread of the 2019-nCoV outbreak originating in Wuhan, China: a modelling study. The Lancet, 395(10225), 689-697. https://doi.org/10.1016/S0140-6736(20)30260-9

Yuksel, B., \& Ozgor, F. (2020). Effect of the COVID-19 pandemic on female sexual behavior. International Journal of Gynecology \& Obstetrics, 150(1), 98102. https://doi.org/10.1002/ijgo.13193

Yuksel, B., \& Ozgor, F. (2020). Effect of the COVID-19 pandemic on female sexual behavior. International Journal of Gynecology \& Obstetrics, 150(1), 98102. https://doi.org/10.1002/ijgo.13193

Zomalheto, Z., Assogba, C., \& Dossou-yovo, H. (2021). Impact of the severe acute respiratory syndrome coronavirus 2 (SARS-CoV2) infection and disease2019 (COVID-19) on the quality of life of rheumatoid arthritis patients in Benin. The Egyptian Rheumatologist,43(1), 23-27. https://doi.org/10.1016/j.ejr.2020.07.001 